\title{
Cytocompatibility and mechanical properties of surgical sealants for cardiovascular applications
}

\author{
Mark H. Murdock, BSc, ${ }^{a}$ Jordan T. Chang, BA, ${ }^{a}$ Samuel K. Luketich, MS, ${ }^{b}$ Drake Pedersen, BSc, \\ George S. Hussey, PhD, ${ }^{a}$ Antonio D'Amore, $\mathrm{PhD},{ }^{\mathrm{b}}$ and Stephen F. Badylak, MD, PhD, DVM ${ }^{\mathrm{a}}$
}

\section{ABSTRACT}

Objectives: The present study compared physical, mechanical, and biologic characteristics of 4 clinically available surgical sealants for cardiovascular repair.

Methods: BioGlue (Cryolife Inc, Kennesaw, Ga), PreveLeak (Mallinckrodt Pharmaceuticals, St Louis, Mo), Tridyne VS (BD, Franklin Lakes, NJ), and Coseal (Baxter Healthcare Corporation, Westlake Village, Calif) were compared for the following properties: hydrated swelling, cytocompatibility, burst strength, biaxial stretching (elasticity), and in vitro degradation.

Results: Sealants showed a wide range of swelling upon hydration. By gravimetric and volumetric measurement, swelling was greatest for Coseal followed by Tridyne VS, BioGlue, and PreveLeak. Tridyne VS was the most cytocompatible based on Alamar Blue assay results, supporting 85\% cell survival compared with $36 \%$ to $39 \%$ survival with the other sealants. All sealants withstood pressure above mean arterial pressure $(70-110 \mathrm{~mm} \mathrm{Hg})$ and physiologic systolic blood pressure (90-140 mm Hg) in an ex vivo arterial flow burst model; lowest peak pressure at failure was PreveLeak at $235 \pm 48 \mathrm{~mm} \mathrm{Hg}$, and highest peak pressure at failure was BioGlue at $596 \pm 72 \mathrm{~mm} \mathrm{Hg}$. Biaxial tensile testing showed no differences in elasticity between ex vivo porcine aorta and carotid arteries and Tridyne VS or Coseal, and BioGlue and PreveLeak were significantly stiffer. In vitro degradation time for Coseal was 6 days and 21 days for Tridyne VS. No degradation was observed in BioGlue or PreveLeak for 30 days.

Conclusions: Although all sealants withstood supraphysiologic arterial pressure, there were differences in characteristics that may be important in clinical outcome. Coseal degradation time was short compared with other sealants, whereas BioGlue and PreveLeak showed a significant compliance mismatch with native porcine carotid artery. Tridyne VS was significantly more cytocompatible than the other 3 sealants. (J Thorac Cardiovasc Surg 2019;157:176-83)

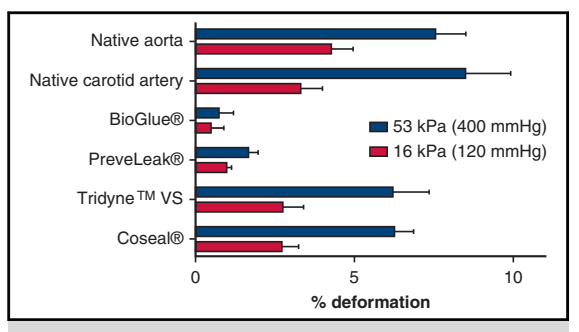

Cardiovascular sealants should possess elasticity similar to cardiovascular tissues.

\section{Central Message}

Surgical sealants for cardiovascular application possess distinct characteristics with respect to swelling, cytocompatibility, strength, elasticity, and degradation rate.

\section{Perspective}

There are several surgical sealants available for use in cardiovascular surgery. Sealant compositions differ with respect to swelling, cytocompatibility, mechanical strength, elasticity, and degradation rate, all of which may affect efficacy and downstream healing. The present study compared the characteristics of 4 sealants used for cardiovascular applications.

See Editorial Commentary page 184.

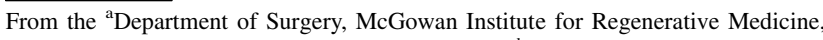
University of Pittsburgh, Pittsburgh, Pa; and ${ }^{\mathrm{b}}$ Departments of Surgery and Bioengineering, McGowan Institute for Regenerative Medicine, University of Pittsburgh, Pittsburgh, Pa.

This work was supported by the National Institutes of Health TL1TR001858 (to M.H.M.), RiMED Foundation 0057091 (to S.K.L. and A.D.), and American Heart Association 17PRE33680043 (to D.P.). Partial funding of this study was provided by BD (Franklin Lakes, NJ).

Received for publication April 26, 2018; revisions received June 18, 2018; accepted for publication Aug 23, 2018; available ahead of print Sept 28, 2018

Address for reprints: Stephen F. Badylak, MD, PhD, DVM, 450 Technology Drive, Bridgeside Point II, Suite \#300, Pittsburgh, PA 15219 (E-mail: badysx@upmc. edu).

$0022-5223 / \$ 36.00$

Copyright (c) 2018 by The American Association for Thoracic Surgery

https://doi.org/10.1016/j.jtcvs.2018.08.043
}

Blood loss during cardiovascular surgery and in the postoperative period is a significant cause of morbidity and mortality. ${ }^{1-3}$ Therefore, it is common to reinforce sutures or staples with surgical sealants to minimize bleeding. ${ }^{3,4}$ Application of such sealants has been shown to effectively control localized bleeding during surgery, reduce the percentage of patients requiring blood products intraoperatively, and reduce postoperative bleeding. ${ }^{5-7}$ One study found that patients who receive blood products during cardiovascular surgery experience a $132 \%$ higher total cost of care, a $156 \%$ longer length of hospital stay, and increased mortality rate compared with patients undergoing cardiovascular surgery who did not receive blood products. ${ }^{8,9}$ Therefore, sealants that effectively 

Abbreviations and Acronyms
BSA = bovine serum albumin
HMEC $=$ human microvascular endothelial cell
IFU $=$ instructions for use
MAP $=$ mean arterial pressure
PBS = phosphate-buffered saline
PEG = polyethylene glycol
PTFE = polytetrafluoroethylene
SBP = systolic blood pressure
$\mathrm{UBM}=$ urinary bladder matrix

\section{MATERIALS AND METHODS Overview of Study Design}

BioGlue, PreveLeak, Tridyne VS, and Coseal were characterized for swelling, cytocompatibility, burst strength, biaxial stretch, and degradation time, and the results were compared among test articles. All sealant samples were prepared per each manufacturer's instructions for use (IFU). All tests used $\mathrm{N}=6$ unless specified otherwise.

\section{Swelling}

Sealants were cast into a $15-\mathrm{mm}$ diameter and 2-mm thick custom polytetrafluoroethylene (PTFE) mold and allowed to polymerize for at least the minimum time indicated in the manufacturer's IFU. Swelling was assessed both gravimetrically by weighing mass with an M-220D analytic balance (Denver Instrument, Bohemia, NY) and volumetrically by measuring diameter and thickness with CD-8"CSX digital calipers (Mituyoto, Kawasaki, Japan). Sealant plugs were placed in $50 \mathrm{~mL}$ Falcon tubes (Fisher Scientific, Hampton, NH) with $20 \mathrm{~mL}$ phosphate-buffered saline (PBS) and $0.02 \%$ sodium azide, and the tubes were then placed in a $37^{\circ} \mathrm{C}$ water bath with gentle agitation. Mass, diameter, and thickness were measured at 0,24 , and 48 hours. Sealants were gently blot dried with paper towel before weighing. Volume was calculated by using the equation volume $=\pi(1 / 2 \mathrm{~d})^{2} \mathrm{t}$, where $t$ is the thickness of the plug and $d$ is the diameter. Mass and volume change are represented by percent change with respect to the time 0 measurements.

\section{Cytocompatibility}

Unpolymerized sealant components and degradation/diffusion products of polymerized sealants were evaluated for cytocompatibility with an immortalized line of human microvascular endothelial cell (HMEC) 1. Unpolymerized sealant components were mixed with HMEC-1 culture media (MCDB-131 media supplemented with $10 \%$ fetal bovine serum, $10 \mathrm{mmol} / \mathrm{L}$ glutamine, and $1 \%$ penicillin/streptomycin) in a 1:3 (v:v) ratio. Polymerized sealants were incubated in media in a $1: 3(\mathrm{v}: \mathrm{v})$ ratio at $37^{\circ} \mathrm{C}$ for 7 days to yield degradation/diffusion products. For the control group, PBS was added to media in a 1:3 (v:v) ratio. HMEC-1, an immortalized HMEC cell line acquired from American Type Culture Collection (Manassas, Va), was plated at a density of $10,000 \mathrm{cells} / \mathrm{cm}^{2}$ in culture media. Cell viability was measured by following the IFU of the alamarBlue Cell Viability Assay (Thermo Fisher Scientific, Inc, Waltham, Mass), which indicates metabolic mitochondrial activity in cells. Cells grew for 24 hours before treatment. Twenty-four hours after treatment, media/treatment was removed and fresh media with $10 \%$ alamarBlue, and without test article was added to each well. Cells were incubated overnight in alamarBlue, and fluorescence was measured at an excitation wavelength of $530 \mathrm{~nm}$ and emission wavelength $590 \mathrm{~nm}$

\section{Burst Testing}

Sealant burst strength was determined by 2 methods. The first method involved sealing a defect in a flat lyophilized sheet of urinary bladder matrix (UBM). UBM is a biologic surgical mesh material composed of the basement membrane and decellularized tunica propia of porcine bladders (Animal Biotech Industries, Danboro, Pa). The second method involved sealing a defect in porcine carotid arteries (Animal Biotech Industries) harvested within 10 minutes after animals were killed and measuring peak pressure at failure. Burst tests were conducted following the American Standard Test Method F2392-04(2015) titled "Standard Test Method for Burst Strength of Surgical Sealants." 12 Following manufacture's IFU, sealants were applied onto a 2-mm defect in a sterilized UBM sheet contained in a custom 15-mm diameter, 2-mm deep PTFE mold. PBS was infused using a PHD2000 pump (Harvard Apparatus, Holliston, Mass) at a flow rate of $5 \mathrm{~mL} / \mathrm{min}$. Pressure was recorded using a 07356-61 pressure transducer (Cole-Parmer, Vernon Hills, Ill), 
DAQ USB-6009, and Labview 8.6 (National Instruments, Austin, Tex). In vitro carotid artery burst tests were conducted on 3- to 7-mm diameter, 12- to 16-cm length porcine carotid arteries. Arteries were secured to male Leur Integral Lock rings using 3-0 polyglycolic acid sutures. Defects were created with an $18 \mathrm{G}$ needle. One milliliter of sealant was applied over each defect per manufacturer's IFU $(\mathrm{N}=5)$. If failure occurred at the interface of the substrate and the sealant (adhesive failure), the test was excluded; only cohesive failures (PBS bursting through the sealant) were recorded.

\section{Biaxial Testing}

Porcine carotid artery and descending aorta were obtained from Animal Biotech Industries (Danboro, Pa). Biaxial mechanical testing of the porcine vessel $(\mathrm{N}=5)$ and surgical sealants $(\mathrm{N} \geq 4)$ was completed as previously described. ${ }^{13}$ The vessel tissue was split longitudinally and trimmed to $10 \times 10$-mm squares, and the 4 test articles were cast between 2 flat plates at a height of $2 \mathrm{~mm}$ and trimmed to $12 \times 12$-mm squares. Thickness was measured at 5 locations (center and 4 corners) using a dial micrometer (Starrett, Athol, Mass) before hydrating in PBS. Four hooks per side were attached $1 \mathrm{~mm}$ from each edge of the specimen, and 4 polypropylene (Ethicon, Somerville, NJ) markers were affixed in a $4 \times 4$-mm square at the center of the specimen to track the deformation tensor gradient during loading and unloading. Each specimen was then placed in PBS at room temperature and loaded using Lagrangian equi-stress control to a maximum stress of $60 \mathrm{kPa}$ or until hook retention failure. The protocol for preconditioning and testing consisted of ten 15-second cycles. Marker free-float position after preconditioning was used as reference for postprocessing in MATLAB (MathWorks, Natick, Mass).

\section{Degradation Time}

Time to degradation was measured by the following standard: American Standard Test Method F1635-16, "Standard Test Method for In Vitro Degradation Testing of Hydrolytically Degradable Polymer Resins and Fabricated Forms for Surgical Implants." ${ }^{14}$ Briefly, sealants were cast in a 2.5-cm diameter, 1-cm deep cylindrical PTFE mold and placed in 50-mL Falcon tubes (Fisher Scientific, Hampton, NH) with $20 \mathrm{~mL}$ PBS and $0.02 \%$ sodium azide. Tubes were placed in a $37^{\circ} \mathrm{C}$ water bath with gentle agitation. Tubes were examined daily for 30 days, and if no sealant was visually discernable, the sealant was considered completely degraded.

\section{Statistical Analysis}

Statistical analyses were performed using a 1-way ordinary analysis of variance (GraphPad Prism 7, La Jolla, Calif) followed by post hoc Tukey's multiple comparisons test.

\section{RESULTS}

All values are reported as mean \pm standard deviation.

\section{Sealant Swelling}

Sealants showed a wide range of swelling values. The following sealants increased in mass after 24 hours (Figure $1, A)$ : BioGlue $(5.98 \% \pm 1.21 \%$ ), Tridyne VS $(53.91 \% \pm 5.85 \%)$, and Coseal $(117.57 \% \pm 10.23 \%)$. PreveLeak decreased in mass $(-2.26 \pm 0.38 \%)$. The following sealants increased in mass after 48 hours (Figure 1, A): BioGlue $(11.38 \% \pm 2.33 \%)$, Tridyne VS $(81.81 \% \pm 9.61 \%)$, and Coseal $(205.32 \% \pm 11.14 \%)$. PreveLeak increased in mass between 24 and 48 hours but was still less than the $\mathrm{t}=0$ mass $(-1.34 \% \pm 0.64 \%)$.
All sealants increased in volume after 24 hours (Figure 1, B): BioGlue $(25.65 \% \pm 3.6 \%)$, PreveLeak $(4.48 \pm 1.68 \%)$, Tridyne VS $(62.18 \pm 10.25 \%)$, and Coseal $(155.79 \% \pm 14.80 \%)$. All sealants increased in volume after 48 hours from time 0 volume (Figure 1, B): BioGlue (36.00 $\pm 4.22 \%)$, PreveLeak $(5.09 \% \pm 2.47 \%)$, Tridyne VS $(84.92 \% \pm 12.01 \%)$, and Coseal $(247.26 \% \pm 24.41 \%)$.

\section{Cytocompatibility}

Unpolymerized components of Tridyne VS showed higher cytocompatibility with HMEC-1 than the other sealants (Figure 2, A). There was no significant difference between the survival of HMEC-1 in the media control compared with cells given unpolymerized Tridyne VS $(P=.70)$. Significantly fewer cells survived treatment with unpolymerized BioGlue, PreveLeak, and Coseal compared with the media control or the unpolymerized Tridyne VS treatment $(P<.0005, P<.005$, Figure $2, A)$. Degradation/diffusion products of PreveLeak and Tridyne VS showed higher cytocompatibility with HMEC-1 than the other sealants (Figure 2, B). HMEC-1 survival in the media control was significantly higher than all sealant treatments $(P<.0001)$. However, significantly more cells survived when treated with PreveLeak and Tridyne VS than BioGlue and Coseal $(P<.0001)$. There were no significant differences in the cytocompatibility between PreveLeak and Tridyne VS or between BioGlue and Coseal (Figure 2, B).

\section{Burst Testing}

The burst strength values with flat UBM sheets and ex vivo carotid arteries showed that no sealants were as strong as the no-defect controls, but all exhibited burst strength well above mean arterial pressure (MAP) and systolic blood pressure (SBP) (Figure 3, $A$ and $B$ ). Peak burst strength through sealants formed on a defect in a UBM sheet are as follows (Figure 3, A): sheets with no defect $(655.81 \pm 43.61 \mathrm{~mm} \mathrm{Hg})$, sheets with defect and no sealant $(3.49 \pm 1.77 \mathrm{~mm} \mathrm{Hg})$, BioGlue $(516.68 \pm$ $93.53 \mathrm{~mm} \mathrm{Hg}$ ), PreveLeak (314.67 $\pm 45.36 \mathrm{~mm} \mathrm{Hg}$ ), Tridyne VS (304.32 $\pm 50.39 \mathrm{~mm} \mathrm{Hg})$, and Coseal $(355.42 \pm 45.50 \mathrm{~mm} \mathrm{Hg})$. BioGlue showed a significantly higher burst pressure than PreveLeak, Tridyne VS $(P<.0001)$, and Coseal $(P<.005)$, and there were no significant differences among the other groups. In the ex vivo arterial burst test, the mean burst strength for artery without defect was $916.58 \pm 57.49 \mathrm{~mm} \mathrm{Hg}$, $12.08 \pm 2.74 \mathrm{~mm} \mathrm{Hg}$ for the defect only control, $596.05 \pm 71.52 \mathrm{~mm} \mathrm{Hg}$ for BioGlue, $234.6 \pm 47.84 \mathrm{~mm}$ $\mathrm{Hg}$ for PreveLeak, $345.2 \pm 115.95 \mathrm{~mm} \mathrm{Hg}$ for Tridyne VS, and $343.00 \pm 92.71 \mathrm{~mm} \mathrm{Hg}$ for Coseal (Figure 3, B). BioGlue was significantly stronger than PreveLeak 

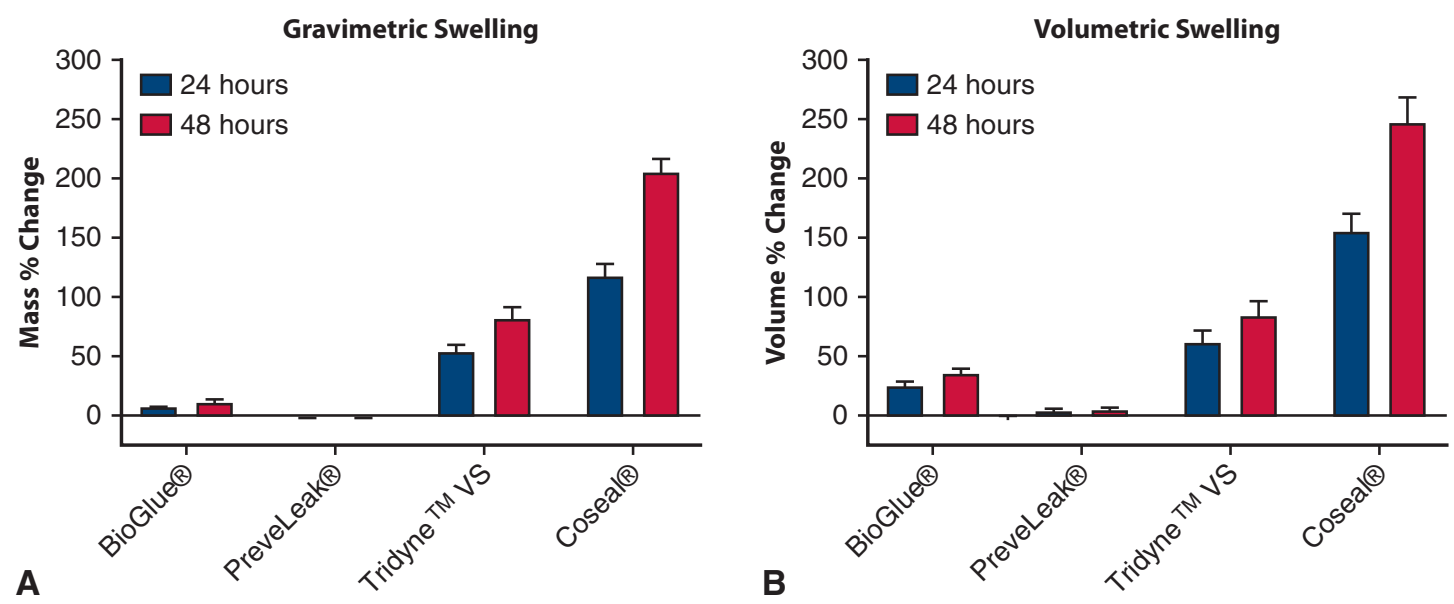

FIGURE 1. Cardiovascular surgical sealants possess drastically different swelling properties. Gel swelling analysis by both gravimetric (A) and volumetric (B) methods at both 24 and 48 hours. Each gel has a distinct swelling profile ranging from virtually no swelling to doubling in mass/size. Data shown as mean \pm standard deviation (SD), $\mathrm{N}=6$.

$(P<.0001)$, Coseal, and Tridyne VS $(P<.005)$, and there were no significant differences among the other groups.

\section{Biaxial Testing}

Biaxial equi-stress testing of the porcine vessels and surgical sealants showed varying degrees of compliance (Figure $4, A$ and $B$ ) up to $60 \mathrm{kPa}$. The peak stresses of Tridyne VS and Coseal were $57 \mathrm{kPa}$ and $53 \mathrm{kPa}$, respectively, as both experienced hook retention failure beyond these peak stress values. Statistical analysis was conducted on data at both $53 \mathrm{kPa}(400 \mathrm{~mm} \mathrm{Hg})$ and $16 \mathrm{kPa}(120 \mathrm{~mm} \mathrm{Hg}$ ) (Figure 4, B). At both values, as expected, the circumferential and longitudinal directions of the native vessel tissue showed different stress responses for the equi-stress load. Tridyne VS and Coseal test articles showed a stress response similar to that of the native tissue. Neither axis was significantly different than the longitudinal or circumferential directions of the native tissue. In contrast, at both values BioGlue and PreveLeak were significantly stiffer than both directions of the porcine carotid artery $(P<.05)$. BioGlue was significantly stiffer than both directions of the porcine descending aorta, whereas PreveLeak was only significantly stiffer than the longitudinal direction $(P<.05)$. Both BioGlue and PreveLeak axes were significantly stiffer than axis 1 of Tridyne VS and Coseal $(P<.05)$.
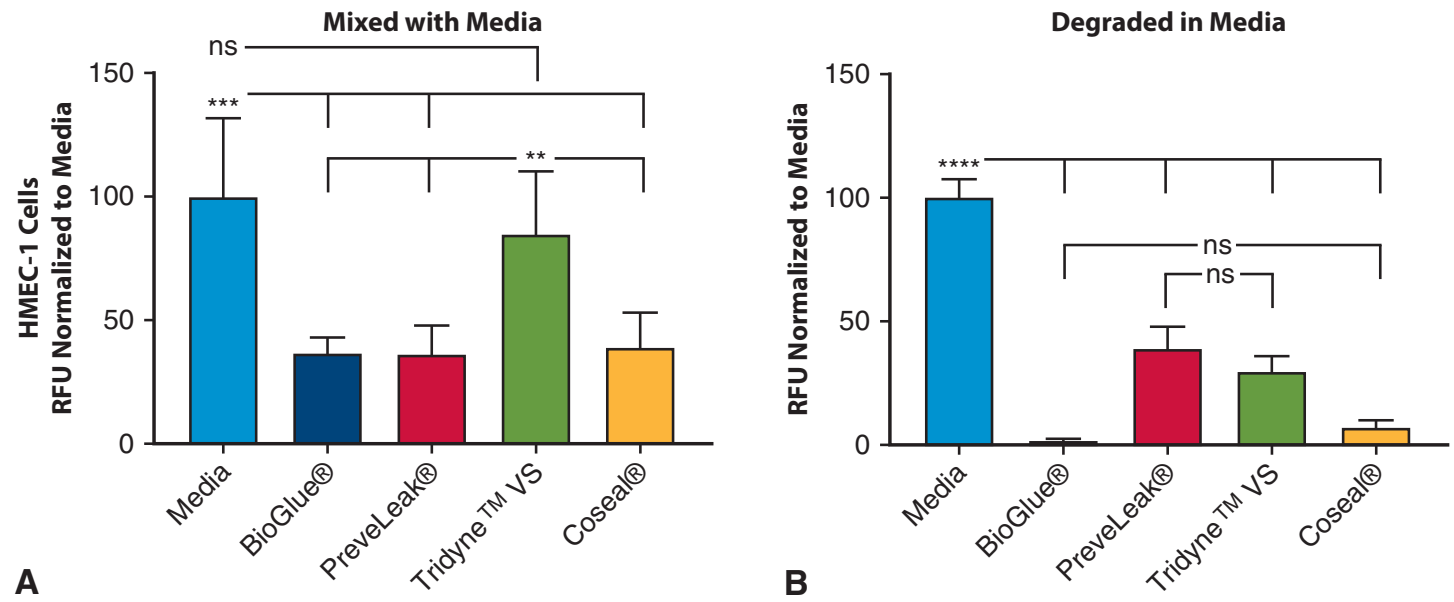

FIGURE 2. Cardiovascular surgical sealants differ in cytocompatibility. Relative fluorescence units from MTT assay of HMEC cells 24 hours after exposure to sealant mixed with media (A) or polymerized sealant degraded in media for 14 days (B), normalized to the media control. There is no significant cytocompatibility difference between predegradation Tridyne VS (BD, Franklin Lakes, NJ) and media, but the degradation products of all gels are significantly lower than the media control. Data shown as mean $\pm \mathrm{SD}, \mathrm{N}=6$. $* * P<.005, * * * P<.0005$. HMEC-1, Human microvascular endothelial cell $1 ; R F U$, relative fluorescence unit. 

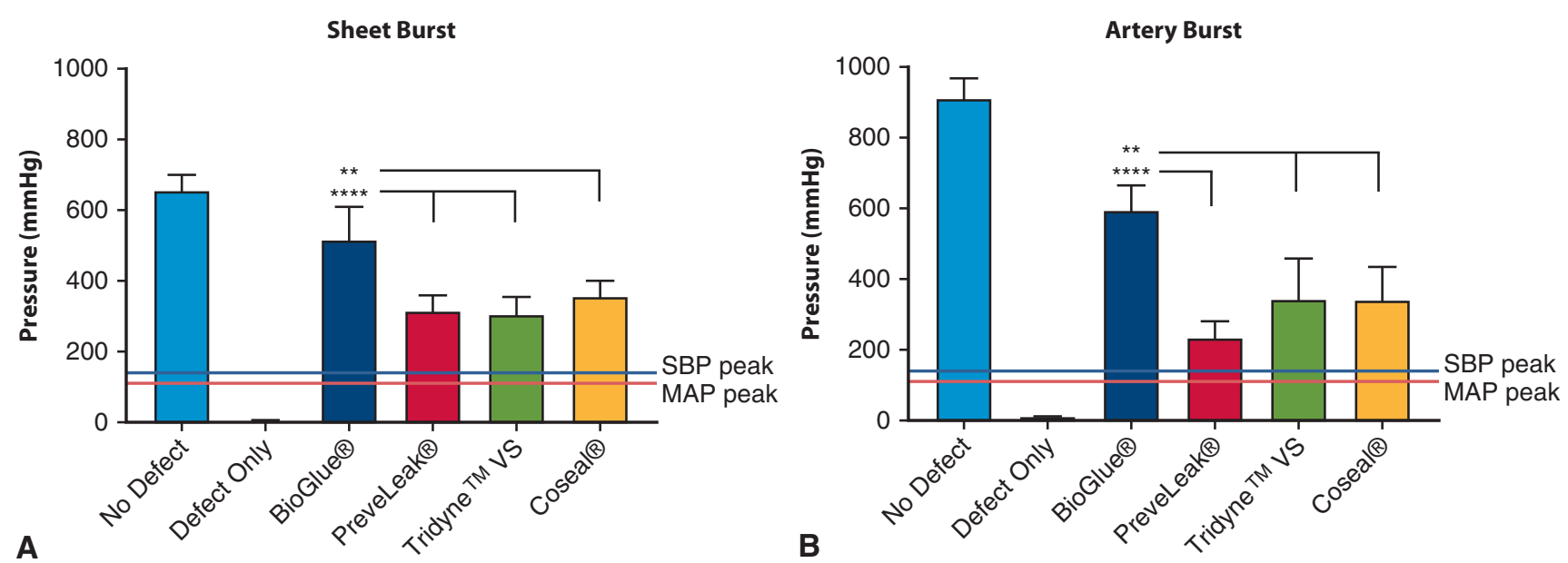

FIGURE 3. Cardiovascular surgical sealants have burst strength above physiologic loads. Burst strength measured by peak pressure through sealant covering a defect on a collagen sheet (A) or through sealant covering a defect in a carotid artery (B). All gels have burst strength higher than MAP, with BioGlue (Cryolife Inc, Kennesaw, Ga) having significantly higher burst strength than other gels. Data shown as mean $\pm \mathrm{SD}, \mathrm{N}=6 . * * P<.005$. $* * * * P<.00005$. MAP peak indicates $110 \mathrm{~mm} \mathrm{Hg}$, and SBP peak indicates $140 \mathrm{~mm} \mathrm{Hg}$. SBP, Systolic blood pressure; MAP, mean arterial pressure.

\section{Degradation Time}

Mean time to degradation was 6 days for Coseal and 21 days for Tridyne VS. BioGlue and PreveLeak showed no macroscopic signs of degradation within 30 days (Table 1).

\section{DISCUSSION}

The present study identifies the similarities and differences among 4 commonly used surgical sealants with respect to clinically relevant physiologic, mechanical, and biologic properties (Table 2 shows sealant compositions ${ }^{15-18}$ ). This study focused primarily on cardiovascular applications, but the results lend insight into their use in soft tissues throughout the body. Gravimetric and volumetric assessment of swelling indicate to what degree a sealant can further increase in mass or volume postapplication. This information may be relevant for use in a confined space, material ability to appose disjoined tissue, or utility in preventing leakage from surgical sites. ${ }^{19}$ It is not surprising that BioGlue and PreveLeak both showed minimal swelling given the tightly knit nature of BSA cross-linking by aldehydes. Despite Tridyne VS and Coseal both being
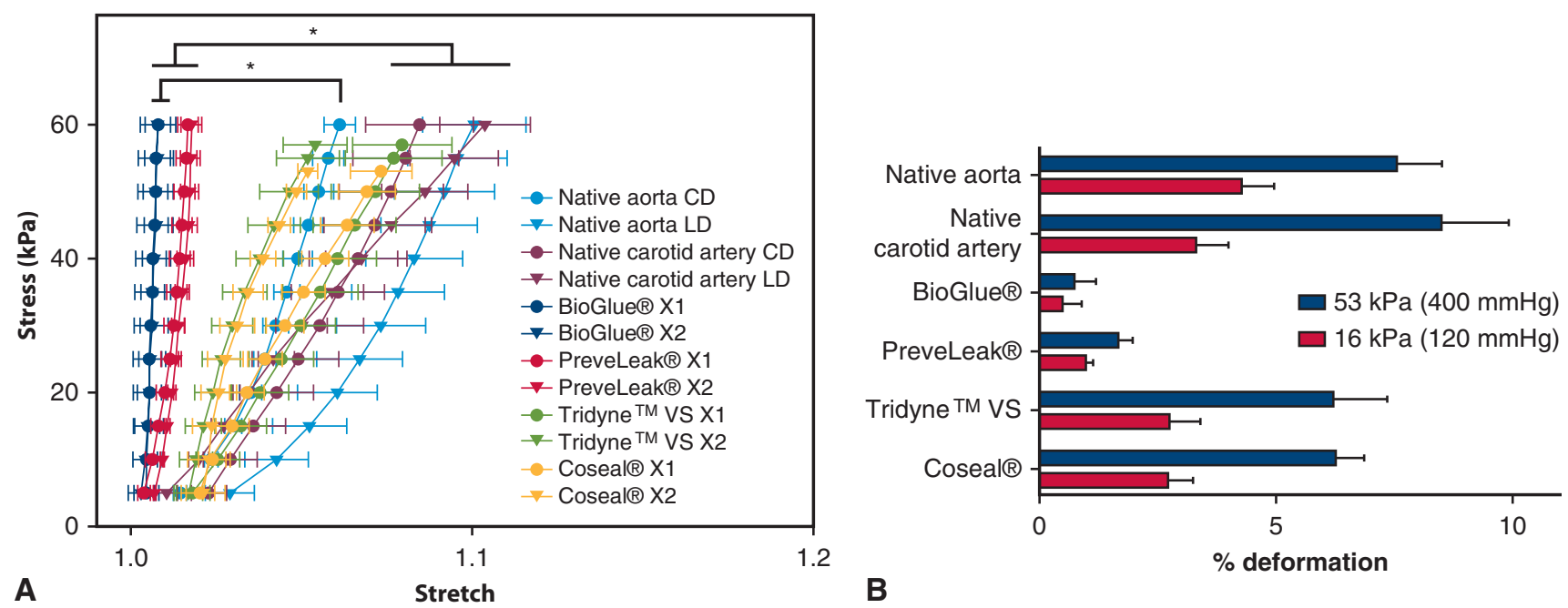

FIGURE 4. Cardiovascular surgical sealants differ in elasticity. Elasticity of sealants was measured biaxially as stress applied to the sample ( $Y$-axis $)$ and subsequent stretch observed (X-axis) and compared with porcine native aorta and carotid artery (A). Statistical analysis was conducted on data at both $53 \mathrm{kPa}$ $(400 \mathrm{~mm} \mathrm{Hg})$ and $16 \mathrm{kPa}(120 \mathrm{~mm} \mathrm{Hg})$ to represent both supraphysiologic and physiologic forces (B). Tridyne VS and Coseal (Baxter Healthcare Corporation, Westlake Village, Calif) have elasticity similar to aorta and carotid artery, whereas BioGlue and PreveLeak (Mallinckrodt Pharmaceuticals, St Louis, Mo) are significantly stiffer. Data plots represent mean $\pm \mathrm{SD}, \mathrm{N}=5$. $C D$, Circumferential direction; $L D$, longitudinal direction. 
TABLE 1. Cardiovascular surgical sealants may degrade slowly or rapidly

\begin{tabular}{lc}
\hline \multicolumn{1}{c}{ Sealant } & Degradation time (d) \\
\hline BioGlue & $>30$ \\
PreveLeak & $>30$ \\
Tridyne VS & 21 \\
Coseal & 6 \\
\hline
\end{tabular}

Sealant plugs were immersed in agitated PBS at $37^{\circ}$ until complete degradation was observed or until 30 days. Coseal fully degraded at 6 days, Tridyne VS degraded at 21 days, and BioGlue and PreveLeak showed no signs of degradation even at 30 days. Data shown represent $\mathrm{N}=3$, representative of 3 independent experiments.

PEG-based polymers, their swelling behavior was markedly different; specifically, Coseal swelled almost twice as much as Tridyne VS. Of note, these swelling behaviors correlate well with the degradation data; the high-density crosslinking of the BSA-based sealants result in longer degradation time, whereas the intermediate-swelling Tridyne VS degraded at an intermediate time and the highly swelling Coseal degraded rapidly.

In assessing the cytocompatibility of sealant composition, it should be noted that reagents may not be completely reacted in the polymerization process causing an excess of 1 reagent. Thus, it is logical to test the sealant's effect on cell viability in the unpolymerized state. Upon application and polymerization, the sealant may be degraded by hydrolysis resulting in the formation of degradation products. HMEC- 1 is an immortalized HMEC that retains the morphologic, phenotypic, and functional characteristics of native HMEC. ${ }^{20}$ Because endothelial cells will be present in cardiovascular and virtually all other anatomic applications of the sealants, HMEC represent a logical choice of cell type for evaluation of cytocompatibility. Given their composition, it is not surprising that unpolymerized BioGlue and PreveLeak exhibited poor cytocompatibility with HMEC-1. Although they are composed largely of purified natural BSA, their aldehyde cross-linkers confer a low cytocompatibility with HMEC-1. It is generally understood that aldehydes are locally cytotoxic and can continue to be released from treated materials for long periods of time. ${ }^{21}$ In fact, cytotoxicity may partially explain the adverse events sometimes reported in association with excessive application of BioGlue, including anastomotic pseudoaneurysms, ${ }^{22-24}$ anastomotic stenosis,${ }^{25}$ pulmonary artery rupture, ${ }^{26}$ and secondary coagulopathy. ${ }^{27,28}$ However, it is surprising that after polymerization, PreveLeak shows better cytocompatibility than BioGlue, possibly because of the use of an unnamed polyaldehyde cross-linker in PreveLeak versus glutaraldehyde in BioGlue. ${ }^{21}$ Tridyne VS and Coseal are both PEG-based materials; PEG hydrogels have shown good cytocompatibility in several tissues, ${ }^{29,30}$ explaining the high cytocompatibility their unpolymerized components have with HMEC-1 (Figure 2, $A)$. However, once Coseal is polymerized, cytocompatibility decreases (Figure 2, B), possibly as a result of the high degree of swelling and subsequent rapid degradation. PEG is a polymer of the toxic monomer ethylene glycol; it is possible that one of the PEGs that make up Coseal degrade into this toxic monomer unit. The PEG contained in Tridyne VS does not contain ethylene glycol in its degradation profile and may result in nontoxic low molecular weight esters or dimer/trimer units of ethylene glycol. $^{31}$

Burst tests used both a flat hydrated UBM sheet, composed mainly of collagen, as well as ex vivo carotid arteries. The UBM sheets were of uniform thickness and avoided the tissue variability that is inherent in ex vivo testing. The carotid arteries more closely represent the in vivo conditions in terms of tubular geometry and adhesion properties. In both methods of testing, all sealants were able to contain pressure well above MAP range and $\mathrm{SBP}$, even for an individual with high blood pressure, with BioGlue showing significantly higher peak burst pressure than the other sealants. This observation is

TABLE 2. Sealant information per instructions for use

\begin{tabular}{|c|c|c|}
\hline Sealant & Composition & Polymerization information \\
\hline $\begin{array}{l}\text { BioGlue (Cryolife Inc, } \\
\text { Kennesaw, Ga) }\end{array}$ & Purified BSA and glutaraldehyde & $\begin{array}{l}\text { "BioGlue works optimally when it is allowed to polymerize } \\
\text { without any manipulation for a full } 2 \text { min." } 14\end{array}$ \\
\hline $\begin{array}{l}\text { PreveLeak (Mallinckrodt } \\
\text { Pharmaceuticals, } \\
\text { St Louis, Mo) }\end{array}$ & Purified BSA and polyaldehyde & $\begin{array}{l}\text { "PreveLeak is applied as a viscous liquid that gels within } \\
\text { approximately } 10-15 \mathrm{sec} . . \text { leave the clamps in place for } \\
\text { at least } 60 \mathrm{sec} \text { before restoring circulation, applying irrigation, } \\
\text { blotting with gauze or touching the sealant."15 }\end{array}$ \\
\hline $\begin{array}{l}\text { Tridyne VS (BD, } \\
\text { Franklin Lakes, NJ) }\end{array}$ & PEG and HSA & "Tridyne VS will form a flexible hydrogel 2 min after application." ${ }^{16}$ \\
\hline $\begin{array}{l}\text { Coseal (Baxter } \\
\text { Healthcare Corporation, } \\
\text { Westlake Village, Calif) }\end{array}$ & $\begin{array}{l}2 \text { synthetic PEGs, a dilute hydrogel } \\
\text { chloride solution, and a sodium } \\
\text { phosphate/sodium carbonate solution }\end{array}$ & $\begin{array}{l}\text { "After application wait at least } 60 \mathrm{sec} \text { before restoring circulation, } \\
\text { applying irrigation, blotting with gauze, or touching the sealant." } 17\end{array}$ \\
\hline
\end{tabular}

Sealant compositions and polymerization information are listed as declared by the manufacturers of each material and accompanying IFU. BSA, Bovine serum albumin; $P E G$, polyethylene glycols; $H S A$, human serum albumin. 
consistent with the high-density crosslinking of BSA by glutaraldehyde, producing a strong sealant membrane. It has been established that polymeric hydrogel adhesives intended for use as arterial vascular sealants should withstand pressures of at least $200 \mathrm{~mm} \mathrm{Hg}$, a metric met by all of these sealants. ${ }^{32}$ In summary, all sealant formulations are capable of containing physiologic blood pressure in the heart and other anatomic locations.

The nature of cardiovascular tissue demands a sealant with high elasticity or compliance. ${ }^{33,34}$ Applied sealants that are too stiff may detach from the application site over time, increasing frequency and severity of postoperative bleeding. The finding that BioGlue and PreveLeak are significantly stiffer than native tissue is not surprising, given the high-density crosslinking of BSA by aldehydes. This increased stiffness was macroscopically appreciable when handling these sealant samples. The 2 PEG-based formulations, Tridyne VS and Coseal, were more compliant, suggesting they are more likely to remain at their original site of application as the heart continues beating. These observations complement the swelling data; it is reasonable that gel materials able to hydrate will become less dense and more compliant than materials that are dense and compact.

The preferred degradation rate for a sealant depends on the anatomic site of application and the severity of the injury. Sealants should not degrade so quickly as to compromise hemostasis, but should not remain at the site of injury long enough to hinder the healing process. Wound resolution in different tissues follows the same general pattern of hemostasis (seconds to hours), inflammation (hours to days), repair (days to weeks), and remodeling (weeks to months), depending on the severity of the injury and the tissue in question. ${ }^{35}$ The degradation assay used in this study provided relative, not absolute, degradation rates of the sealants. In vivo, enzymatic or cellular activity in addition to thermal/mechanical factors would increase the degradation rate. In addition, degradation data provided were dependent on mass, surface area to mass ratio, and geometry of the bulk sealants tested. The plugs used in this study were cylinders of $2.5 \mathrm{~cm}$ diameter and $1 \mathrm{~cm}$ depth, equating to $4.9 \mathrm{~mL}$ of sealant, that is, probably more than would be used for any single instance of hemostasis. With these stipulations in mind, Tridyne VS takes approximately 3.5 times longer to degrade than Coseal. The assay used complete dissolution of a material as the end point to measure time to degradation. The assay did not extend beyond 30 days; therefore, the amount of BioGlue or PreveLeak that degraded during this time cannot be determined. However, it is known that BioGlue remains in humans for at least 9 months after application and has not been observed to degrade or resorb in rats for up to 12 months. ${ }^{33}$ Taken together, it is likely that clinically relevant amounts of Coseal would degrade quicker than
6 days, that BioGlue and PreveLeak will require up to 1 year to degrade, and that Tridyne VS may persist at the site of application for several weeks.

\section{CONCLUSIONS}

The different formulations of surgical sealants confer unique physicochemical properties that should be considered when selecting a preferred hemostatic sealant for cardiovascular or vascular surgery. Cyanoacrylate sealants were not evaluated in this study because they are known to have high stiffness, low elasticity, and high toxicity in areas with significant moisture, making them a poor candidate for cardiovascular hemostasis. Two natural polymer-based sealants were assessed: BioGlue and PreveLeak. BioGlue had the lowest cytocompatibility with HMEC-1, but the highest burst strength (all sealants held pressure well above MAP and SBP). PreveLeak had the second best cytocompatibility with HMEC-1. BioGlue and PreveLeak both exhibited almost no hydrated swelling, which was associated with elasticity lower than native aorta/carotid tissue and degradation rate in the scale of months to years. Tridyne VS showed the best cytocompatibility with HMEC-1, moderate swelling that was associated with high elasticity, and a degradation rate on the scale of weeks. Coseal exhibited the third best cytocompatibility with HMEC-1 and a high degree of swelling that was associated with high elasticity and a degradation rate on the scale of days. Although in vitro and ex vivo systems do not perfectly reflect in vivo conditions, the results reported may provide insight that can be combined with clinical experience and existing literature to inform sealant selection. Surgical sealants have distinct physical, mechanical, and biologic characteristics that should be considered before application in any anatomic location, but particularly for challenging sites such as cardiovasculature.

\section{Conflict of Interest Statement}

S.F.B. receives research funding from BD, Franklin Lakes, New Jersey. All other authors have nothing to disclose with regard to commercial support.

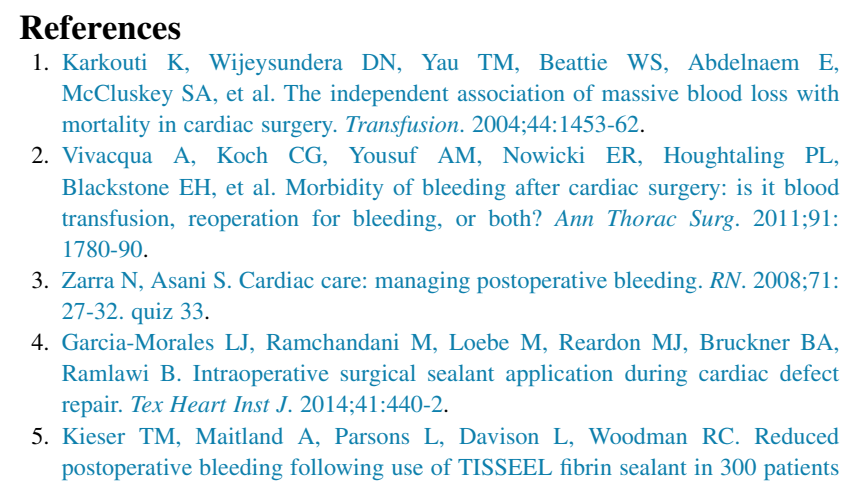


undergoing open-heart surgery. In: Schlag G, Wolner E, Eckersberger F, eds. Fibrin Sealing in Surgical and Nonsurgical Fields. Berlin, Germany: Springer; 1995:43-54.

6. Rousou J, Levitsky S, Gonzalez-Lavin L, Cosgrove D, Magilligan D, Weldon C, et al. Randomized clinical trial of fibrin sealant in patients undergoing resternotomy or reoperation after cardiac operations. A multicenter study. J Thorac Cardiovasc Surg. 1989;97:194-203.

7. Nasso G, Piancone F, Bonifazi R, Romano V, Visicchio G, De Filippo CM, et al. Prospective, randomized clinical trial of the FloSeal matrix sealant in cardiac surgery. Ann Thorac Surg. 2009;88:1520-6.

8. Zbrozek A, Magee G. Cost of bleeding in trauma and complex cardiac surgery. Clin Ther. 2015;37:1966-74.

9. Arias-Morales CE, Stoicea N, Gonzalez-Zacarias AA, Slawski D, Bhandary SP, Saranteas T, et al. Revisiting blood transfusion and predictors of outcome in cardiac surgery patients: a concise perspective. F1000Res. 2017;6:168.

10. Kazemzadeh-Narbat M, Annabi N, Khademhosseini A. Surgical sealants and high strength adhesives. Materials Today. 2015;18:176-7.

11. Bot GM, Bot KG, Ogunranti JO, Onah JA, Sule AZ, Hassan I, et al. The use of cyanoacrylate in surgical anastomosis: an alternative to microsurgery. J Surg Tech Case Rep. 2010;2:44-8.

12. ASTM F2392-04. Standard Test Method for Burst Strength of Surgical Sealants. West Conshohocken, PA: ASTM International; 2015. Available at: www.astm. org. Accessed December 16, 2016.

13. Sacks MS. Biaxial mechanical evaluation of planar biological materials. J Elast. 2000;61:199.

14. ASTM F1635-16. Standard Test Method for in vitro Degradation Testing of Hydrolytically Degradable Polymer Resins and Fabricated Forms for Surgical Implants. West Conshohocken, PA: ASTM International; 2016. Available at: www.astm.org. Accessed December 16, 2016.

15. BioGlue [package insert]. Kennesaw, GA: Cryolife; 2014.

16. PreveLeak [package insert]. Mountain View, CA: The Medicines Company; 2016.

17. Tridyne [package insert]. Warwick, RI: C.R. Bard; 2017.

18. Coseal [package insert]. Hayward, CA: Baxter Health Corporation; 2009.

19. Shazly TM, Baker AB, Naber JR, Bon A, Van Vliet KJ, Edelman ER. Augmentation of post-swelling surgical sealant potential of adhesive hydrogels. J Biomed Mater Res A. 2010;95:1159-69.

20. Ades EW, Candal FJ, Swerlick RA, George VG, Summers S, Bosse DC, et al. HMEC-1: Establishment of an immortalized human microvascular endothelial cell line. J Investig Dermatol. 1992;99:683-90.

21. Marinucci L, Lilli C, Guerra M, Belcastro S, Becchetti E, Stabellini G, et al. Biocompatibility of collagen membranes crosslinked with glutaraldehyde or diphenylphosphoryl azide: an in vitro study. J Biomed Mater Res A. 2003;67: 504-9.

22. David TE. False aneurysms after heart valve surgery. J Thorac Cardiovasc Surg. 2017:153:51-2.

23. Luk A, David TE, Butany J. Complications of BioGlue postsurgery for aortic dissections and aortic valve replacement. J Clin Pathol. 2012;65:1008-12.

24. Kobayashi T, Kurazumi H, Sato M, Gohra H. Pseudoaneurysm rupture after acute Type A dissection repair: possible reaction to BioGlue. Interact Cardiovasc Thorac Surg. 2017;26:331-2.

25. Gundry SR, Black K, Izutani H. Sutureless coronary artery bypass with biologic glued anastomoses: preliminary in vivo and in vitro results. J Thorac Cardiovasc Surg. 2000;120:473-7.

26. Ma WG, Ziganshin BA, Guo CF, Zafar MA, Sieller RS, Tranquilli M, et al Does BioGlue contribute to anastomotic pseudoaneurysm after thoracic aortic surgery? J Thorac Dis. 2017;9:2491.

27. Streiff MB, Ness PM. Acquired FV inhibitors: a needless iatrogenic complication of bovine thrombin exposure. Transfusion. 2002;42:18-26.

28. Poynton AR, Nelson MC, McCance SE, Levine RL, O'Leary PF. Bovine thrombin induces an acquired coagulopathy in sensitized patients undergoing revision spinal surgery: a report of two cases. Spine. 2003;28:E221-3.

29. Bjugstad KB, Lampe K, Kern DS, Mahoney M. Biocompatibility of poly(ethylene glycol)-based hydrogels in the brain: an analysis of the glial response across space and time. J Biomed Mater Res A. 2010;95A:79-91.

30. Alcantar NA, Aydil ES, Israelachvili JN. Polyethylene glycol-coated biocompatible surfaces. J Biomed Mater Res. 2000;51:343-51.

31. Han S, Kim C, Kwon D. Thermal/oxidative degradation and stabilization of polyethylene glycol. Polymer. 1997;38:317-23.

32. Ghobril C, Grinstaff MW. The chemistry and engineering of polymeric hydrogel adhesives for wound closure: a tutorial. Chem Soc Rev. 2015;44: 1820-35.

33. Annabi N, Zhang YN, Assmann A, Sani ES, Cheng G, Lassaletta AD, et al Engineering a highly elastic human protein-based sealant for surgical applications. Sci Transl Med. 2017;9:eaai7466.

34. Annabi N, Yue K, Tamayol A, Khademhosseini A. Elastic sealants for surgical applications. Eur J Pharm Biopharm. 2015;95:27-39.

35. Stroncek JD, Reichert WM. Overview of wound healing in different tissue types. In: Reichert WM, ed. Indwelling Neural Implants: Strategies for Contending with the In Vivo Environment. 1st ed. Boca Raton, FL: CRC Press; 2008:3-41.

Key Words: sealant, compatibility, pressure 